KOINONIA. Revista Arbitrada Interdisciplinaria de Ciencias de la Educación, Turismo, Ciencias Sociales y Económica, Ciencias del Agro y Mar y Ciencias Exactas y

Nicolás Pablo Barrientos Oradini; Luis Araya Castillo; Fernando Alejandro Herrera Ciudad; Natalie del Carmen Muñoz Morales

\title{
Actitud docente y apropiación tecnológica en educadores de nivel medio de la región metropolitana de Chile
}

Teaching attitude and technological appropriation in the environment of the metropolitan region of Chile

Nicolás Pablo Barrientos Oradini

nbarrientoso@docente.uss.cl

Universidad San Sebastián

Chile

https://orcid.org/0000-0002-5854-9501

Luis Araya Castillo

luis.arayac@uautonoma.cl

Universidad Autónoma de Chile

Chile

https://orcid.org/0000-0001-7574-3907

Fernando Alejandro Herrera Ciudad

fernando.herrera@profe.umcervantes.cl

Universidad Miguel de Cervantes

Chile

https://orcid.org/0000-0002-7141-7335

Natalie del Carmen Muñoz Morales

natalie.munoz@profe.umcervantes.cl

Universidad Miguel de Cervantes

Chile

https://orcid.org/0000-0002-7079-3099

Recibido: 10 de octubre del 2018

Aprobado: 09 de diciembre del 2018 


\section{RESUMEN}

El presente artículo se propone analizar la actitud docente y apropiación tecnológica en instituciones de educación media. Para dar cumplimiento con dicho propósito se realizó una revisión de la literatura relativa a las variables objeto de estudio. Se enmarcó como un estudio descriptivo, de campo, transeccional y no experimental. La población estuvo estructurada por treinta y cuatro (34) docentes de instituciones educativas de la región metropolitana de Chile, los cuales poseen características similares ya que se desempeñan en sus labores académicas en instituciones de educación media. Para la recolección de la información, se utilizó una encuesta diseñada con escalamiento tipo Lickert, conformada por treinta (30) ítems. Se evidenció problemáticas diversas, demostrando la capacidad de los equipos de trabajo docente para fortalecer y potenciar el desarrollo tecnológico en los estudiantes de las unidades educativas objeto de estudio.

Descriptores: Actitud del docente; tecnología; percepción; motivación; satisfacción en el trabajo.

\section{ABSTRACT}

The present article intends to analyze the teaching attitude and technological appropriation in secondary education institutions. In order to comply with this purpose, a review of the literature related to the variables under study was carried out. It was framed as a descriptive study, of field, transectional and not experimental. The population was structured by thirty-four (34) teachers from educational institutions in the metropolitan region of Chile, which have similar characteristics since they work in their academic work in secondary education institutions. For the collection of information, a survey designed with Lickert type escalation, consisting of thirty (30) items, was used. Various problems were evidenced, demonstrating the inability of the teaching teams to strengthen and enhance the technological development of the actors of the educational community under study.

Descriptors: Teaching attitude; technology; perception; motivation; satisfaction at work.

\section{INTRODUCCIÓN}

En el contexto de la sociedad del conocimiento, a fines de la primera década del siglo XXI, según lo expuesto por Romaní (2008), se han incrementado los discursos que plantean que la revolución tecnológica demanda repensar las metodologías de enseñanza y extender la penetración de los nuevos dispositivos de información y comunicación en el entorno educativo. 
Sin embargo, son las nuevas formas de generar, organizar y difundir el conocimiento, a raíz de la incorporación de una oferta tecnológica de bajo costo, lo que ha impulsado a la sociedad a integrar nuevas apropiaciones sociales de las tecnologías de información y comunicación (TIC).

En el actual contexto, se desarrollan nuevas formas de interacción en tiempos y escenarios, dejando de ser los docentes espectadores, para convertirse en protagonistas de la información en un mundo donde el intercambio de ideas y de culturas son actos permanentes a través de las TIC.

Lo anteriormente mencionado se ve reforzado por lo expuesto por la Organización de las Naciones Unidas para la Educación, la Ciencia y la Cultura (UNESCO, 1998) citada en (Diago, 2013), quien señala el impacto de las TIC en las técnicas convencionales de enseñanza y de aprendizaje, auspiciando también la transformación en su proceso y la forma en que tanto docentes como alumnos accedan al conocimiento y a la información.

Entre las muchas estrategias que aportan las TIC'S para que tanto docentes como alumnos accedan al conocimiento y a la información, se encuentran los recursos educativos abiertos (REA) que, según Gutiérrez (2010), son innovaciones que consiste en materiales que se pueden compartir con instituciones de cualquier parte del mundo.

Al respecto, Adegbija (2009), se refiere a la actitud del docente como una disposición a reaccionar favorable o desfavorablemente hacia una situación o suceso. Para ello es necesario que las instituciones educativas estén atentas a identificar aquellos asuntos que acontecen en el docente como sujeto, a sus emociones y al impacto que las nuevas tecnologías pueden tener en los procesos que emprende para implementarlas en el aula.

Sobre el particular, existe un plan de acción regional en América latina que aborda el fomento de políticas educativas orientadas al desarrollo del conocimiento por medio de las Tecnología de información. El plan regional representa el compromiso de los gobiernos de los países latinoamericanos de avanzar hacia sociedades de la 
información. Organización de las Naciones Unidas para la Educación, la Ciencia y la Cultura (Unesco 2018).

Una de las principales estrategias a implementar, en opinión de los investigadores, es la motivación a los docentes en las TIC, por cuanto son ellos quienes inducen y transfieren los conocimientos al alumnado. Se observa que los jóvenes se integrarán cada vez más naturalmente y ampliamente en el mundo de la tecnología, pero ello genera nuevas brechas de distanciamiento con los demás, que van acumulándose y superponiéndose, haciendo que la distancia sea cada vez más irreconciliable.

Por ende, las políticas orientadas a las TIC, deben avanzar más allá de la incorporación de equipamiento tecnológico en las escuelas, que sigue siendo muy relevante para algunos países de la región, e ir hacia la apropiación tecnológica orientada a los docentes instruyendo a los mismos para su función, porque los estudiantes necesitan orientación para aun buen uso y aprovechamiento de las herramientas tecnológicas.

En ese sentido, los docentes mantienen un rol protagónico en las escuelas, con un papel principal en la construcción de una sociedad moderna, viable y exitosa, fundamentalmente basada en las TIC, como mecanismos de interconexión con su entorno.

Cabe considerar entonces que las TIC ofrecen la posibilidad de interacción que pasa de una actitud pasiva por parte de los alumnos a una búsqueda y replanteamiento incesante de contenidos y procedimientos, desarrollando su iniciativa en las tareas, ya que se ven obligados continuamente a tomar decisiones, filtrar información, escogerla y socializarla; situación que en algunos casos se ve entorpecida por docentes que no asumen su rol de orientador dentro del aula.

No obstante, de acuerdo a este planteamiento, para que en la educación se puedan explorar los beneficios de las TIC en el proceso de aprendizaje, es esencial que, tanto los futuros docentes como los que están en actividad, sepan manejar estos instrumentos y para lograrlo es necesario educarse y actualizarse, lo que implica un esfuerzo para adaptarse a una nueva forma de ver la educación que pareciera no ser 
asumido por los docentes, en algunos casos por desinterés, por hostilidad al cambio, y a la tecnología.

Sin embargo, el personal docente en los niveles de educación media que fueron objeto de estudio en Chile, suelen hacer poco uso de la tecnología para el desarrollo de los procesos de aprendizaje en el aula. Algunas de las limitaciones es la resistencia a procesos de actualización que permita mejorar las debilidades en el uso de las herramientas tecnológicas (Computador, Tablet, iPod, etc) y por ende obtener los beneficios que ellas traen consigo.

Es por ello que a través de ésta investigación se busca analizar la actitud docente y apropiación tecnológica en instituciones de educación media, de manera que los mismos puedan promover el desarrollo métodos y con ello la adquisición de una actitud favorable hacia la transferencia de conocimientos logrando que los alumnos se sientan motivados en los distintos procesos de adquisición del conocimiento. Igualmente, se pretende proponer lineamientos teóricos para la aplicación de los REA como mecanismo de apropiación tecnológica en la mencionada institución.

\section{DESARROLLO}

\section{Actitud docente}

En lo que respecta al tema, según Amorós (2007) la actitud es un tipo de diferencia individual que afecta el comportamiento de las personas, especialmente relativa a creencias orientadas a agrupar ideas, temáticas determinadas. Para Nicoll, (2018) la actitud puede definirse de modo formal como una disposición supuestamente aprendida a reaccionar de alguna manera característica ante un estímulo particular. Las actitudes no son innatas, sino que se adquieren y por lo tanto son susceptibles de ser modificadas y educadas, desde la escuela se puede intervenir en este sentido, aunque se trate de disposiciones relativamente estables.

De lo expuesto, se deduce que Amorós (2007) realiza una definición previa de lo que actitud abarca, así como Nicoll, D. (2018) plantean una percepción similar de la actitud 
siendo esta positiva o negativa dependiendo de si se enfrenta a un objeto, situaciones o personas. Es así que se acoge el criterio expuesto por el autor Nicoll, D. (2018) siendo este amplio en detalles facilitando la realización de una conceptualización propia. En resumen, la actitud docente es el comportamiento equilibrado del docente dentro del salón de clases y que permite el éxito de los escolares y del docente.

\section{Componentes afectivo de la actitud docente}

La afectividad se define según Foster Marín y Rojas-Barahona (2008) como todos los eventos y acontecimientos emocionales que acontecen en la mente de los hombres y que se reflejan mediante un comportamiento emocional. En este sentido, el autor citado, argumenta que el componente afectivo está constituido por el ámbito afectivo emocional.

Por otro lado, agrega López (2012) que es la sensación y sentimientos que dicho objeto produce en el sujeto. Es decir, el sujeto puede experimentar distintas experiencias con el objeto, estas pueden ser positivas o negativas.

Al respecto, Vargas (2009) plantea que la afectividad condiciona la actividad del individuo, de tal forma que entre el pensamiento y la acción se interpone siempre lo afectivo, moderando o estimulando los actos, siendo la conducta condicionada por la afectividad.

Afirmando entonces que el docente se presenta tal como su afectividad lo condiciona, debido a que su actitud afectiva tiene un origen instinto-afectivo que va a manifestarse en forma placentera o no, en cada uno de los seres humanos, frente a los procesos físicos, ambientales y psíquicos; por lo que la influencia de la afectividad es tan amplia que puede modificar el entorno ambiental.

Lo anteriormente planteado es reforzado por Neisser (2014) quien afirma que el entorno agradable, favorece positivamente todo proceso de aprendizaje, y Prendes (2011) reafirma que, la actitud afectiva que el docente asume durante su actividad diaria funcionará como modelo de estimulación, capaz de producir en el alumno ciertas 
respuestas, que orientarán la acción académica hacia un aprendizaje significativo. Al respecto Gallego (2010) plantea, que todo ser humano posee una gran capacidad para la formación de actitudes afectivas, consideradas como un factor esencial para responder a los estímulos y que en oportunidades la afectividad y emoción se utilizan de manera intercambiable e incluyen sentimientos, de allí que la afectividad determina la actitud general del ser humano.

Según Amorós (2007), el componente afectivo se da en tres etapas principales que debe tomar en cuenta el docente para su desenvolvimiento emocional midiendo así la actitud, estas etapas consisten en: percepción, motivación y satisfacción del trabajo, siendo estas etapas acogidas por la presente investigación y desarrolladas a continuación.

En resumen, Foster Marín y Rojas-Barahona (2008) proporcionan las bases para la conceptualización de lo que comprende el componente afectivo, por su parte López (2012) comenta respecto a la afectividad mientras que Neisser (2014) y Prendes (2011) ya hacen mención de dicha afectividad en el entorno educativo.

Ahora bien, Vargas (2009) expresa que las actitudes afectivas son formadas por toda persona, siendo Amorós (2007) quien proporciona una clasificación de los componentes afectivos. En base a lo expresado por los autores antes citados, se puede inferir que el componente afectivo requiere la reacción del sujeto hacia un objeto o actividad determinada, y las reacciones a cada uno de los atributos que este pueda poseer.

\section{Percepción.}

Según Vargas y Gamboa, (2016), es el conjunto de sensaciones, completadas, corregidas e interpretadas por el sujeto con la ayuda de sus experiencias, para generar conocimientos de hechos, objetos o fenómenos reales. También se puede definir como un proceso de mediación en el que intervienen la sensación y la conducta, en ella intervienen las características de los estímulos que activan los órganos, a experiencia del sujeto, su motivo, su actitud, su personalidad total. 
Por otra parte, según la psicología clásica de Neisser (2014), la percepción es un proceso activo-constructivo en el que el perceptor, mucho antes que la información pueda ser procesada y guardada en la conciencia, se va construyendo el estructura informativa anticipatoria, que facilita poder contrastar el estímulo de manera que se pueda aceptar o rechazarlo en función a su adecuación a la propuesta en el estructura esquemática. La percepción de un individuo es subjetiva, selectiva y temporal.

Al respecto, Robbins y Coulter (2014) argumenta que la mayoría de las personas reconocen que las percepciones tienen influencia sobre el aprendizaje. Todo estudiante ha experimentado el impacto de sus percepciones en relación con el docente, otros alumnos, hasta de sus propias habilidades y el valor de las tareas asignadas.

Ahora bien, Vargas y Gamboa, (2016) y Neisser (2014) realizan una descripción general de lo que comprende la percepción, siendo que Robbins y Coulter (2014) quienes realizan el enlace de la percepción en la educación, siendo esta una influencia en el aprendizaje. En base a los postulados, se determina la conceptualización aportada por el autor antes citado para la presente investigación y se deduce que cuando las percepciones para con el docente son positivas, el aprendizaje se optimiza.

\section{Motivación.}

La motivación se entiende como un proceso general por el cual se inicia y dirige una conducta hacia el logro de una meta. De ella, se refiere Robbins y Judge (2015) mencionan que que dicho proceso implica la consideración de aspectos cognitivos como afectivos. Involucra variables afectivas, en tanto comprende elementos como la autovaloración, auto concepto, entre otros.

De la misma manera, Hogg (2010) señala que, si se realiza una revisión de las teorías de la motivación, se observa que destacan los siguientes constructos: el auto concepto, los patrones de atribución causal, y las metas de aprendizaje. En consecuencia, estos factores y su interrelación determinarán en gran medida la motivación. Por ello, se considera que la motivación constituye uno de los factores psicoeducativos que más 
influyen en el aprendizaje.

De acuerdo con Amorós (2007), se refiere a las fuerzas que actúan sobre un individuo o en su interior, y originan que se comporte de una manera determinada, dirigida hacia las metas, condicionado por la capacidad del esfuerzo de satisfacer alguna necesidad individual. Debido a que los motivos para desarrollar un trabajo por parte de los empleados influyen en la productividad, se constituye en una de las tareas de los gerentes encaminar efectivamente la motivación del empleado, hacia el logro de las metas de la organización.

Se observa que los autores poseen distintos criterios, Hogg (2010) así como Robbins y Judge (2015) la perciben como un proceso para alcanzar metas en el aprendizaje, su doctrina está dirigida al ámbito de los procesos de aprendizaje-enseñanza. Por otro lado, Amorós (2007) ofrece una definición adaptable y general de lo que abarca la motivación, sirviendo ello para ser acogida por la presente.

Los procesos motivacionales, implican estados internos que direccionan a la persona al logro de metas y objetivos específicos; estos estados se constituyen como impulsos que potencian e impulsan a las personas a tomar algunas acciones y comportamientos y mantener persistencia hasta logra las metas.

Igualmente, se considera como fuerzas que se originan intrínsecamente en las personas y que originan comportamientos que inciden en los entornos laborales.

\section{Satisfacción en el trabajo.}

Según Kinicki y Kreitner (2012), "la satisfacción en el trabajo es una respuesta emocional y afectiva de la persona a diversos aspectos del trabajo que realiza". (p.154). De acuerdo a los autores, esta acepción implica que la satisfacción adquiere cierta relatividad, en el sentido que una persona puede estar muy satisfecho con algunos aspectos laborales, sin embargo, muestra insatisfacción con otros, creando complejidades especialmente en los entornos de la gestión de la educación por la implicancia que tiene la insatisfacción del docente ante los alumnos. 
Asimismo, Robbins (2009, p.181), define la satisfacción laboral, centrándose básicamente, en los niveles de satisfacción e insatisfacción sobre la proyección actitudinal de positivismo o negativismo, definiéndola como "la actitud general de un individuo hacia su trabajo. Una persona con un alto nivel de satisfacción en el puesto tiene actitudes positivas hacia el mismo; una persona que está insatisfecha con su puesto tiene actitudes negativas hacia él".

Amorós (2007) por su parte, define satisfacción del trabajo de manera muy genérica, como la actitud general de la persona hacia su trabajo, en el sentido que las actividades que toda persona realiza en entornos de trabajo van más allá que simples asignaciones, implican relaciones interpersonales entre colegas y con diferentes niveles de la organización.

Por otra parte, y según lo expresa el autor citado, incluye las políticas, así como el cumplimiento de las normas y los estándares de rendimiento laboral, e incluso con las condiciones de trabajo. Por lo anteriormente citando, la evaluación de la satisfacción de un empleado llega a ser la sumatoria de varios aspectos que le dan cierta complejidad.

Así mismo el autor Amoros (2007), agrega que la satisfacción en el trabajo es una actitud hacia el trabajo de uno, la diferencia entre la cantidad de recompensas que los empleados reciben y la cantidad que ellos consideran debe recibir.

Ahora bien, Kinicki y Kreitner (2012) y Robbins (2008) ofrecen unos postulados basados en la satisfacción laboral del trabajador, mencionando importantes detalles en relación a ello. Sin embargo, en razón de los criterios expuestos, los investigadores del presente estudio consideran la teoría mencionada por Amorós (2007) en razón de que describe de manera acertada el sentido que la investigadora desea dar al tópico, describiéndolo como una actitud hacia el trabajo de uno. Desde el aporte del autor citado, la satisfacción es un objetivo propio de la organización, no solo está relacionada de manera negativa con el ausentismo y la rotación sino que también las organizaciones deben brindar a sus empleados labores desafiantes e intrínsecamente recompensables. 


\section{Componentes conductuales de apropiación tecnológica}

Según Robbins y Judge (2015), es una variable latente, que ha de ser inferida de ciertas respuestas mensurables y que reflejan en última instancia, una evaluación global positiva o negativa del objeto de la actitud. De forma similar, Corral (2003) citado por Pérez y Rodríguez (2014), señalan que el componente conductual son las intenciones, disposiciones o tendencias hacia un objeto, es cuando surge una verdadera asociación entre objeto y sujeto.

Igualmente Hellriegel, (2009), manifiesta que los comportamientos del ser humano se manifiestan producto de la construcción de conocimientos aprendidos y desarrollados. De allí que se desprende que las habilidades conductuales crecen con el comportamiento social, son reciprocas por naturaleza y suponen una correspondencia afectiva adecuada.

En ese mismo orden Hernández, S. (2011), expresa que la práctica de las habilidades sociales está influenciada por las características del medio, por ejemplo, factores como la edad, sexo, estatus social, entre otros.

De acuerdo con Betancourt y Valadez (2012), los componentes conductuales se comprenden como aquellas habilidades que muestra una persona en expresar sus sentimientos positivos como negativos, en diferentes contextos donde se dan las relaciones interpersonales, de tal forma que disminuyan los diferentes conflictos que pudieran estar asociados en ellas.

Para los investigadores, el componente conductual envuelve una evaluación de un objeto o persona que tienen una dirección que las hace experiencia agradable 0 desagradable. En esa misma dirección Alvariño y Severín (2009), sostienen que mantienen correspondencia con la conducta social; sin embargo, existe consistencia entre la expresión de la actitud y la conducta asociada.

\section{Disposición.}

La disposición en la actitud es adquirida y relativamente duradera a evaluar de una 
manera determinada un objeto, persona, suceso o situación y a actuar en consonancia con la evaluación, ello en base a lo dispuesto por Prat, Flores y Soler (2014). En general, se coincide en que las actitudes solo son una de las determinantes de la conducta, representan, tal como lo sostiene Asinsten, J. (2007), una disposición a comportarse, pero la manera en que se actúa en realidad dentro de una situación en particular, incluso en entornos virtuales de enseñanza.

También dependerá de las consecuencias inmediatas de la conducta, de cómo se piensa que los otros evaluaran estas acciones y de los modos habituales de comportarse en este tipo de situación (Bañuelos Márquez, 2013).

Arroyo (2018), expresa que la disposición es una actitud personal que usualmente está basada en la experiencia, que influye en el comportamiento de un individuo. En otros términos, la disposición puede considerarse como una forma de motivación social o grupal.

Según al autor citado, esta se expresa de carácter emotiva, racional e inconsciente, de allí la complejidad que adquiere la actitud para ser cambiada. Es entonces que desde una visión psicosocial y crítico no pudiera definirse como un proceso de disposición estable, por el contrario, llega a constituirse como una alternativa relativa los discursos que todo contexto cultural permite.

Arroyo (2018) y Prat, Flores y Soler (2014) exponen posturas similares que envuelve la disposición, que según los autores está basada en la experiencia influyendo en el comportamiento de un individuo. Sin embargo, es la postura de Arroyo (2018) la que se acogerá en la presente, en razón de mostrar con mayor detalle el tópico estudiado. Los investigadores del estudio deducen que la disposición es un elemento importante, debido al avance tecnológico y la implementación de TIC en la educación, así que el docente al poseer una disposición positiva enfrenta los cambios donde los individuos adaptándose a ellos, por lo que se debe comenzar en la organización como lo sostiene Marqués, (2012).con una disposición para el cambio de la vanguardia en el proceso educativo. 


\section{Predisposición.}

La predisposición impulsa a actuar de una determinada manera, compuesta de elementos cognitivos (creencia), afectivo (valoraciones) y de conductual (tendencia a responder), siendo ello expuesto por Prat, Flores y Soler (2014). Las predisposiciones estables de la interioridad que el ser humano adquiere a partir de los valores en los que cree, y que lo hacen relacionar o comportarse favorable o desfavorablemente, ante realidades vividas, ideas, situaciones, personas o acontecimientos. Según Hogg (2010) la predisposición a actuar antes de ejecutar un comportamiento o conducta que puede estar influenciado, por algún tipo de componente del carácter personal.

Para Moya, M y Rodríguez R. (2011), la actitud hacia la predisposición es para acercar valoración positiva- o evitar -valoración negativa-, cierta clase de objetos. En ese sentido, las actitudes los autores la conceptualizan como una tendencia o predisposición que es adquirida que puede mostrar el modo en que las personas evalúan los sucesos o las situaciones de la realidad. De allí que, las actitudes se convierten en una herramienta predictora de las conductas.

Las opiniones son expresiones verbales de disposiciones subyacentes. En ese sentido, y tal como lo sostiene Bañuelos, (2011), las opiniones se perciben como expresiones concretas de las actitudes, probablemente sea más apropiado indagar las funciones de las actitudes en sí.

Prat, Flores y Soler (2014), Hogg (2010), así como Moya, M y Rodríguez R. (2011) no diferencian mucho sus conceptualizaciones en cuanto a la predisposición, quedan claro que cada autor aporta un detalle a lo que comprende el concepto.

Desde la perspectiva de los investigadores, la predisposición se inclina hacia algún propósito o alguna situación determinada donde se hace énfasis a una disposición anticipada de la conducta del individuo para un fin concreto, evitando así las tareas presentadas en una determinada actividad o situación. De este modo, según las cualidades psicológicas de una persona, se puede predecir una serie de acontecimientos o circunstancias que tendrán mayor probabilidad de suceder, dada su 
forma de vivir.

\section{Habilidad}

En relación con la habilidad, Robbins S. y Judge T. (2015) manifiestan que las capacidades con las que se nace son la base de las habilidades que después, gracias al aprendizaje y a la experiencia, desarrollará y perfeccionará a lo largo de la vida. Según Amorós (2007), la habilidad desde el punto de vista conductual de la actitud se convierte como la capacidad que el hombre desarrolla para realizar una acción, constituyendo un activo real de todo los que las personas pueden realizar.

En ese sentido la Cepal (2008), ha expresado que no todos poseen habilidades desarrolladas, el hecho de no ser iguales en habilidades, no implica que algunos individuos sean en consecuencia inferior a otros, lo que expresa es que poseen fortalezas y debilidades en términos de la habilidad que los hace ser relativamente superiores o inferiores a los otros, al realizar determinadas tareas o actividades.

Entre las habilidades señaladas por Robbins S. y Judge T. (2015), se puede resaltar la habilidad intelectual, son aquellas que se necesitan en la realización de las actividades mentales, las dimensiones de la actividad intelectual son: La aptitud numérica, la comprensión verbal, la velocidad perceptual, el razonamiento inductivo, el razonamiento deductivo, la visualización especial y la memoria.

Para el punto de la habilidad, según el investigador es el grado de competencia de un sujeto hacia un objeto determinado, ejecutando así la destreza hacia la realización de una cosa, objeto o situación reforzando así la capacidad de conseguir los objetivos a través de un hecho. Generalmente las habilidades comienzan con un infinitivo, enseñamos estimar el alcance del individuo hacia una actividad o enseñanza de algún propósito. 


\section{MATRIZ METODOLÓGICA}

La presente investigación tiene un alcance descriptivo y correlacional, ya que persigue describir como se presenta la actitud docente y la apropiación tecnológica, llegando a considerar la determinación de la relación entre ellas, para ello se realizará el cálculo de la correlación de las variables para analizar las relaciones entre ellas.

La misma se enmarcó en un diseño no experimental, ya que se observan los fenómenos tal y como se dan en la realidad. Igualmente tiene un enfoque cuantitativo, al considerar en el proceso de recolección, ordenamiento y análisis de los datos el uso de la estadística descriptiva y de medida de tendencia central para la interpretación de los resultados. (Hernández, Fernández y Baptista, 2014).

De igual manera, considerando la dimensión temporal, se presenta como un diseño de investigación transeccional o transversal, ya que se recolectaron datos en un solo momento, en un tiempo único. En ese sentido su objetivo es la descripción de las variables en un espacio de tiempo específico, abarcando incluso diferentes grupos de personas u objetos de estudios, realizándose siempre en un momento único.

Por otra parte, tiene un diseño de campo, según Sabino (2009), puesto que los datos se recogen en forma directa de la realidad mediante el trabajo del investigador; es decir, que la información se extrae directamente de una situación real con miras a presentar resultados, y con el fin de optimizar los procesos llevados a cabo en las mismas, es decir, la información se recoge directamente en el propio sitio donde se dan los hechos. La prueba de validez del instrumento de recolección de información asegura que realmente midió lo que se requería medir. En ese sentido, Hernández, Fernández y Baptista (2014), expresan que la validez conceptual respecto a las variables o fenómenos que se pretenden medir, implica la obtención de la medida cuantitativa mediante la cual un cuestionario puede ser aplicado.

Para el presente estudio, la población se estructuró por treinta y cuatro (34) docentes pertenecientes a instituciones educativas, los cuales poseen características similares entre las instituciones analizadas, ya que se desempeñan en labores académicas a 
tiempo completo, sin considerar el género como característica discriminante. Por ser instituciones que mostraron su aprobación a ser consideradas en el estudio la muestra se constituyó como intencional, seleccionando la totalidad de la población como muestra.

Para el proceso de recolección de información se utilizó una encuesta diseñada por los investigadores durante el segundo semestre del año 2017, con escalamiento tipo Lickert, conformada por veinticuatro (24) ítems, que representan las dos dimensiones con tres indicadores cada una y cuatro reactivos. El escalamiento fue con cinco alternativas de respuesta: siempre, casi siempre, a veces, casi nunca y nunca, a los cuales se les asignó un puntaje numerrico de 5, 4,3,2 y 1 respectivamente.

Para su uso se procedió a aplicar una prueba piloto de forma de garantizar la fiabilidad de la misma. El uso de encuesta es un instrumento de recolección de datos comúnmente usado en los estudios de investigación y es decisión propia e los investigadores su aplicación, dándole el carácter científico la confiabilidad estadística calculada.

La validez del mismo fue por juicios de expertos, para lo que se presentó un formato de validación el cual fue entregado a diez (10) expertos. Seguidamente y para garantizar la fiabilidad se aplicó una prueba piloto a 5 docentes de la misma instituciones, de forma de determinar el coeficiente de confiabilidad estadística. La misma se calculó por medio del coeficiente Alfa de Cronbach, utilizando el software estadístico SPSS en su versión 20, arrojando valor de 0,90 para actitud docente y 0,917 para apropiación tecnológica.

\section{RESULTADOS}

Se detallan los resultados con la finalidad de aportar conclusiones y recomendaciones que analice la praxis educativa en aula en las instituciones objeto de estudio, dada la complejidad en sus tareas, y las implicaciones que pudiera conllevar la mala praxis de las acciones ejecutadas en tales centros educativos. 
Para el procesamiento y ordenamiento de los datos recopilados mediante el cuestioanrio utilizado, se utilizó la estadística descriptiva utilizando el software estadistico SPSS en su versión 20,0. En ese sentido, sostiene Arias (2012), que ésta herramienta la emplea el investigador para el procesamiento y ordenameinto de la información, favoreciendo organizar los datos relativos a la variable de estudio.

En este aspecto, los resultados arrojados por el instrumento diseñado por los investigadores que midió la percepción, motivación y la satisfacción al trabajo en la actitud de los docentes de las instituciones objeto de estudio, se presentan en tablas reflejando cada pregunta con sus alternativas de respuestas, de forma absoluta ( $\mathrm{Fa}$ ) y relativa ( $\mathrm{Fr} \%$ ), las cuales permiten determinar en forma clara y precisa el número de repeticiones de las respuestas que arroja una alternativa, además se puede observar el porcentaje que representa cada una de ellas.

Tabla 1. Componente ad la actitud docente

\begin{tabular}{ccccc}
\hline Indicadores & Siempre & $\begin{array}{c}\text { Casi } \\
\text { siempre }\end{array}$ & Casi nunca & Nunca \\
\hline Percepción & $39,22 \%$ & $28,43 \%$ & $19,61 \%$ & $12,75 \%$ \\
Motivación & $38,24 \%$ & $32,35 \%$ & $17,65 \%$ & $11,76 \%$ \\
Satisfacción al & $42,16 \%$ & $26,47 \%$ & $20,59 \%$ & $10,78 \%$ \\
trabajo & & \multicolumn{2}{c}{2,97} & \\
Media & & \multicolumn{2}{c}{2,97} \\
\hline
\end{tabular}

Fuente: elabloración propia

En la Tabla 1 mostrada relativa al análisis de la variable actitud docente, se observa la frecuencia en las respuestas de los docentes consultados en las entidades educativas con mayor tendencia en la opción siempre, pero con porcentajes importantes en la opción casi siempre y en las opciones restantes, reflejados en la tabla; de la cual se evidencia que el indicador Percepción, se ubica en un 39,22\%; el indicador Motivación se ubica en un $38,24 \%$, y el indicador Satisfacción al trabajo refleja una frecuencia en las respuestas de $42,16 \%$, todos ubicados en la opción siempre. 
De la misma manera, se observa el promedio de la media aritmética en 2.97; lo cual ubica tales resultados en la categoría de Medianamente adecuado, y de acuerdo a la selección de las opciones de respuestas otorgadas por los encuestados de forma voluntaria y con el consentimiento por parte de ellos.

Tales resultados fueron deducidos de las respuestas de los encuestados conformados por docentes de educación media en las instituciones que fueron objeto de estudio, niveles bajo en cuanto a los componentes, percepción, motivación y satisfacción en el trabajo, ya que muestran poca capacidad para asumir experiencias ni conocimiento significativo, para construir actividades que estimulen su quehacer; así como tampoco se muestran siempre dispuestos al cumplimiento de metas personales ni institucionales, mostrando actitudes negativas ante los requerimientos de los miembros de la comunidad educativa.

Tabla 2. Componentes apropiación tecnológica

\begin{tabular}{ccccc}
\hline Indicadores & Siempre & $\begin{array}{c}\text { Casi } \\
\text { siempre }\end{array}$ & Casi nunca & Nunca \\
\hline Disposición & $37,25 \%$ & $26,47 \%$ & $20,59 \%$ & $15,69 \%$ \\
Predisposición & $40,20 \%$ & $23,53 \%$ & $21,57 \%$ & $14,71 \%$ \\
Habilidad & $41,18 \%$ & $28,43 \%$ & $17,65 \%$ & $12,75 \%$ \\
Media & \multicolumn{2}{c}{2,91} \\
\hline
\end{tabular}

Fuente: elaboración propia.

En la Tabla 2 mostrada relativa al análisis de la variable apropiación tecnológica, y sus dimensión componentes conductuales, conformados por disposición, predisposición y habilidad, se observan las frecuencias en las respuestas de los docentes consultados, mostrando una mayor tendencia en la opción siempre, pero con porcentajes relevantes en las demás opciones; en la cual se evidencia que la disposición se ubica en un $37,25 \%$, el indicador Predisposición en un $40,20 \%$ y el indicador Habilidad en un $41,18 \%$. 
De la misma manera, se observa el promedio de la media aritmética en 2.91, lo cual ubica tales resultados en la categoría de Medianamente adecuado, dada la escogencia a las opciones de respuestas por parte del personal docente de las organizaciones educativas estudiada.

De igual manera, se evidenciaron que su actuación personal están basadas en sus propias creencias dejándose influenciar por otros; así como en ocasiones se muestra con antivalores en cuanto a su comportamiento docente, evidenciándose negligencia o apatía en las tareas y actividades tecnológicas que debe diseñar para su propio proceso de mejora y la optimización del proceso de aprendizaje de los estudiantes; mostrando, por el contrario, desconocimiento sobre los equipos y dispositivos tecnológicos existentes.

Tabla 3. Correlación entre actitud docente y apropiación tecnológica

\begin{tabular}{|c|c|c|c|}
\hline & & Actitud docente & $\begin{array}{l}\text { Apropiación } \\
\text { tecnológica }\end{array}$ \\
\hline \multirow{3}{*}{ Actitud docente } & $\begin{array}{c}\text { Correlación de } \\
\text { Pearson }\end{array}$ & 1 & , 104 \\
\hline & Sig. (bilateral) & & 346 \\
\hline & $\mathrm{N}$ & 34 & 34 \\
\hline \multirow{3}{*}{ Apropiación tecnológica } & $\begin{array}{c}\text { Correlación de } \\
\text { Pearson }\end{array}$ & 104 & 1 \\
\hline & Sig. (bilateral) & ,346 & \\
\hline & $\mathrm{N}$ & 34 & 34 \\
\hline
\end{tabular}

Fuente: elaboración propia.

Una vez aplicado el cálculo de la correlación mediante el uso del software estadístico SPSS, la misma arrojó un índice de correlación de $r r=0,346$, la cual infiere de acuerdo al baremo se categoriza como una correlación positiva débil, que expresa que en la medida que los valores de la variable actitud docente varía, se produce una variación en la misma dirección de los valores de la variable apropiación tecnológica de forma débil. Expresada esa cantidad al cuadrado arroja el siguiente valor $0,346^{2}=0,12$ que multiplicado por 100 da como resultado el grado de determinación compartido entre ambas variables, lo cual sugiere que la actitud docente y la apropiación tecnológica 
comparten un $12 \%$ de elementos comunes.

\section{Discusión}

En relación a los componentes de la actitud docente, resultados contradicen la teoría de Foster Marín y Rojas-Barahona (2008) quien argumenta que el componente afectivo debe estar constituido por un ámbito afectivo emocional adecuado. Asimismo, contrarían a Amorós (2007), quien realiza la clasificación que él considera para el componente afectivo, la cual debe darse en tres etapas principales que debe tomar en cuenta el docente para su desenvolvimiento emocional midiendo así la actitud, estas etapas consisten en: Percepción, Motivación y Satisfacción del Trabajo.

En este sentido, en las instituciones educativas estudiadas, se requiere que el docente sea capaz de captar todos aquellos estímulos que le permitan su mejora personal y profesional, además de asumir su compromiso ante las metas organizacionales, todo lo cual se traduzca en una actitud positiva reflejada en sus acciones del quehacer académico.

En relación a los Componentes Conductuales los hallazgos contradicen lo expuesto por Robbins y Judge (2015), para quienes los comnponentes conductuales de la actitud es una variable latente, que ha de ser inferida de ciertas respuestas mensurables y que reflejan en última instancia, una evaluación global positiva o negativa del objeto de la actitud.

De forma similar, contradicen los planteamientos de Pérez y Rodríguez (2014), quienes señalan que el componente conductual son las intenciones, disposiciones o tendencias hacia un objeto, es cuando surge una verdadera asociación entre objeto y sujeto.

Del resultados de la correlación mostrada se infiere que en las instituciones educativas que fueron objeto de estudio, la apropiación tecnológica muestra una baja relación con la actitud d elso docentes en los entornos eduactivos.

Es importante señalar lo expresado por Gómez (2018) en relación a fomentar un ambiente que propicie "una cultura de la investigación con el uso de diferentes 
herramientas virtuales $(\ldots)$ orientada por docentes investigadores para fomentar el desarrollo de capacidades y competencias investigativas, cognitivas y sociales que favorezcan la generación de una comunidad de aprendizaje" (p. 77). Siendo este aspecto fundamental para estimular una actitud optima y una mayor motivación en el desarrollo del proceso educativo con los estudiantes.

\section{CONCLUSIONES}

De acuerdo a los resultados del estudio y mostrados anteriormente, los docentes se caracterizan por ser perceptivos, muy motivados y muy satisfecho con la labor docente desarrollada. Igualmente logran desarrollar las habilidades docentes con alta disposición hacia su labor

De lo planteado es posible inferir la necesidad de los docentes pertenecientes a dichas unidades educativa de mantener su compromiso institucional, de desarrollar su sentido de pertenencia, debiendo comportarse favorablemente frente a la tecnología, asumiendo la modernidad y la conveniente implantación de los equipos tecnológicos en aula.

Referente al componente afectivo de la actitud docente en las instituciones educativas en la región metropolitana de Chile, se evidenció que no siempre se perciben estímulos que mejoren su actitud, sus conocimientos, no se muestra motivación a un quehacer académico efectivo a través del uso de las nuevas tecnologías, ante lo cual existe resistencia al cambio lo cual implica insatisfacción, desinterés, apatía en el ejercicio de su labor académica.

En segundo término, acerca del componente conductual de la actitud docente, se logró evidenciar una tendencia baja en cuanto a la disposición para desarrollar las transformaciones educativas, sobre todo en lo atinente a las nuevas tecnologías, ya que se muestra apegado a sus creencias tradicionales, desestimando la actualización para la mejora de sus procesos de aula. 
De las conclusiones obtenidas, se recomienda en general, a los directivos, docentes, y personal administrativo de las instituciones educativas estudiada, la utilización de las nuevas tecnologías de la inmformación y la comunicación, con la finalidad de adherirse a la nueva sociedad tecnológica, con el fin de facilitar los procesos académicos. $Y$ en particular, con respecto a las debilidades encontradas en los docentes se sugiere lo siguiente:

Llevar a cabo programas de capacitación de personal en materia tecnológica, con la finalidad de fortalecer las competencias del personal docente orientadas a afrontar los cambios suscitados en el entorno educativo.

Es necesario llevar a cabo un proceso de cambio liderado por los mismos docentes, mediante prácticas continuas de sensibilización, asumiendo una nueva forma de enseñanza, descartando los enfoques tradicionales memorísticos, generando la disposición de adecuarse a los esquemas requeridos por nuevos paradigmas, e incluso, por las nuevas exigencias estudiantiles.

Ejercitar prácticas interactivas a través del uso de computadores, equipos telefónicos, Internet, pudiendo establecer encuentros didácticos entre ellos, mediante el diálogo y la reflexión entre los docentes que permita la constrastación de la práctica docente con la utilización de las tecnología de información y sus beneficios en el desarrollo del aprendizaje significativo.

Fortalecer las competencias tecnológicas más complejas, utilizando recursos que contengan contenidos educativos esenciales, con la finalidad de utilizar equipos tecnológicos más complejos, además de realizar prácticas para la elaboración de software educativos, portales, páginas Web, y utilizar aplicaciones multimedia diversas que flexibilicen sus esquemas de trabajo y optimicen su planificación educativa.

El estudio tuvo algunas limitaciones en cuanto a la muestra alcanzada. En éste sentido se recomienda a otros investigadores llevar a cabo estudios similares para ampliar las observaciones de la actitud docente y la apropiación tecnologica en ellos. 
De acuerdo a los resultados de la correlación se evidención una relación débil entre la actitud docente y la apropiación tecnológica, en el sentido que en la medida que la primera observe alguna variación en su comportamiento se produce una variación en la segunda de forma débil.

\section{Consideraciones finales}

En líneas generales, los docentes mostraron tener la disposición y la motivación para el desarrollo de competencias en el uso de la tecnología aplicadas a los entornos educativos. Así mismo, las instituciones educativas pudieran aplicar las tecnologías en las aulas de forma de construir aprendizaje significativo. En este sentido el uso de las tecnologías en el aula produciría un nuevo comportamiento en los docentes al permitirse la utilización de otras vías para la transmisión de conocimiento, conduciendo a un aprendizaje significativo en los alumnos.

Los resultados mostraron una coincidencia con las teorías expuestas, evidenciando que el uso de las tecnologías en los procesos educativos produce temáticas para el desarrollo de investigaciones futuras en otros campos de la educación y en diferentes contextos. Sin embargo la no coincidencia de los resultados de la actitud docente con lo expuesto por los autores, hace posible inferir que en dichas instituciones la actitud docente tiene una baja tendencia al uso de la tecnología.

El presente estudio tiene una relevancia para futuras investigaciones al mostrar resultados de la actitud docente y la apropiación tecnológica en instituciones de educación media, permitiendo que desde éstos datos realizar otros estudios que permitan construir nuevos conocimientos en éstas temáticas.

\section{REFERENCIAS CONSULTADAS}

1. Adegbija, S. (2009). Psicología Laboral. México: Editorial Limusa.

2. Alvariño, C. y Severín, E. (2009), Aprendizajes en la Sociedad del Conocimiento. Punto de quiebre para la introducción de las TICs en la educación de América 
Latina. CEPAL. Santiago, Chile.

3. Amorós, E. (2007). Comportamiento Organizacional. Perú.

4. Asinsten, J. (2007). Guía de Trabajo del Docente Contenidista. Producción de Contenidos para la Educación Virtual. Recuperado de https://virtualeduca.org/documentos/manual del contenidista.pdf.

5. Arias, F. (2012) Proyecto de investigación: introducción a la metodología científica ( $4^{\circ}$ ed.) Caracas: Espíteme.

6. Arroyo, S. (2018). Emociones: La cara oculta de las altas capacidades. Editorial Horsori, España.

7. Bañuelos Márquez, A. (2013). Hacia una Docencia Pertinente. Revista Iberoamericana para la Investigación y el Desarrollo Educativo, Num 10, 58-61.

8. Bañuelos, M. A. (2011). La formación docente necesaria en el Siglo XXI. En: J.A. Jerónimo, M. (Coordinador). Educación en Red, nativos digitales y aprendizaje inmersivo UNAM: FES Zaragoza.

9. Betancourt M, J y Valadez M. (2012). Cómo propiciar el talento y la creatividad en la escuela. Editorial Mundo Moderno, México.

10. Cepal (febrero de 2008). Estrategia Latinoamericana y del Caribe de la Sociedad de la Información. En II Conferencia Ministerial sobre la Sociedad de la Información eLAC2007. Conferencia llevada a cabo en San Salvador, El Salvador.

11.Diago, F. (2013). De la Visión a la Acción. Declaración Mundial sobre la Educación Superior. Revista Punto de Vista, IV(5), 173-183.

12. Foster Marín y Rojas-Barahona (2008). Evaluación al interior del aula. Una mirada desde la validez, confiabilidad y objetividad. Revista Pensamiento Educativo, Vol. 43, 295-305.

13. Gallego, M. y otros. (2010). Competencias Digitales en la Formación del Futuro Docente. Propuestas Didácticas. España: Universidad de Granada.

14.Gutiérrez, F. (2010). Implementación de Recursos Educativos Abiertos (REA) a través del portal TEMOA (Knowledge Hub) del Tecnológico de Monterrey, México. Revista Formación Universitaria, 3(5), 9-20. 
15.Gómez ., L. (2018). EXPERIENCIA EN EL DISEÑO DE INSTRUMENTO PARA LA VIRTUALIDAD. Revista Arbitrada Interdisciplinaria Koinonía, 2(3), 65-79. Recuperado http://fundacionkoinonia.com.ve/ojs/index.php/revistakoinonia/article/view/52.

16. Hernández, S. (2011). Apropiación Tecnológica usando Recursos Educativos Abiertos en Proceso de Enseñanza de Ingles. Mexico: Tecnológico de Monterrey EGE.

17. Hernández, Fernández y Baptista (2014). Metodología de la Investigación. McGraw Hill Interamericana. México.

18. Hellriegel, S. (2009) Comportamiento Organizacional. 12va Edición. Cengage Learning. México.

19. Hung, E. (2010). TIC's, Comunicación y periodismo Digital. Tomo II. Escenarios de Enseñanza y Ciberperiodismo. Colombia: Ediciones Uninorte.

20.Hogg V. (2010). Psicología Social. 5ta edición. Madrid: Editorial Médica Panamericana.

21.Kinicki y Kreitner (2012). Comportamiento Organizacional.. México. D.F.: Editorial Paidós.

22. López, M. (2012). Estrategias de Diseño Instruccional para Facilitar el Proceso de Apropiación Tecnológica en Profesores que incorporan Recursos Educativos Abiertos en Educación Virtual. México: Tecnológico de Monterrey EGE.

23. Marqués, P. (2012). Impacto de las TICs en la educación: Funciones y Limitaciones. Revista de Investigación. Num. 1(3), 1-15. Disponible en: http://www.3ciencias.com/wp-content/uploads/2013/01/impacto-de-las-tic.pdf.

24. Moya , M y Rodríguez R. (2011). Fundamentos de la Psicología Social. Editorial Pirámides, España.

25. Neisser, U. (2014). Cognitive Psychology. Psychology Press. EEUU

26. Nicoll, D. (2018). Guía para las pruebas Diagnósticas. México: Editorial McGrawHall.

27.Prat, M., Flores, G. y Soler S. (2014). La voz del profesorado de educación física sobre su formación académica ante la realidad multicultural: análisis de la situación y propuestas de mejora. Revista Electrónica Interuniversitaria de 
Formación del Profesorado. 17(2), 183-199.

28.Prendes, M. y otro (2011). Competencias Tecnológicas del Profesorado de las Universidades Españolas. España: Universidad de Murcia.

29.Prat, M. y Soler, S. (2003). Actitudes, valores y normas en la educación. España: Barcelona.

30.Robbins, S., (2009). Comportamiento organizacional. México. Editorial Pearson Prentice Hall, México.

31. Robbins S. y Judge T. (2015). Comportamiento Organizacional. $15^{\circ}$ Edición, Pearson, México.

32. Robbins S. y Coulter M. (2014). Management. 12Edición, Pearson, México.

33. Romaní, C. (2008). 3er Encuentro de auto-estudio de las Universidades Públicas Mexicanas. Aprendizaje Adaptable y Apropiación Tecnológica: Reflexiones prospectivas. Recuperado en en: http://www.laisumedu.org/DESIN_lbarra/autoestudio3/ponencias/ponencia33.pdf.

34.Sabino, C. (2009). El proceso de Investigación. Venezuela: Editorial Panapo. 2da reimpresión.

35.UNESCO (1998). Declaración mundial sobre la educación superior en el siglo XXI: visión y acción. París. Recuperado de: http://unesdoc.unesco.org/images/0011/001163/116345s.pdf.

36. UNESCO. (2018). Sistema de Información de Tendencias Educativas en América Latina (SITEAL). Obtenido de: http://www.tic.siteal.iipe.unesco.org/.

37. Vargas, B. (2009). El docente y sus actitudes. Colombia. McGraw - Hill.

38. Vargas, G. y Gamboa, R. (2012). El modelo de Van Hiele y la enseñanza de la geometría. Uniciencia, 27 (1), 74-94.

C2019 por los autores. Este artículo es de acceso abierto y distribuido según los términos y condiciones de la licencia Creative Commons Attribution (http://creativecommons.org/licenses/by/4.0/). 